\title{
Tentativas de suicídio em Monte Carmelo-MG
}

\author{
Suicides attempting in Monte Carmelo-MG \\ DOI: 10.5935/2447-8539.20190002
}

\section{ISMELINDA MARIA DINIZ MENDES SOUZA}

e-mail: ismelinda.souza@gmail.com

\begin{abstract}
Resumo
O suicídio é considerado um fenômeno humano, em ascensão, complexo e universal, por ocorrer em todas as sociedades, o que o torna um grave problema de saúde pública, contudo, ele pode ser evitado. 0 objetivo deste estudo foi descrever o perfil das pessoas que tentaram suicídio no município de Monte Carmelo MG. Trata-se de um estudo epidemiológico descritivo cujos dados sobre tentativa de suicídio foram obtidos por meio das fichas de notificação compulsória de intoxicação exógena e violência interpessoal/autoprovocada do SINAN/MS, no período de janeiro a dezembro de 2018. As 78 pessoas que tentaram suicídio são em sua maioria do sexo feminino, com idades entre menores de 20 e 40 anos, com formação até o ensino médio, solteiras, pardas, desempregadas ou sem ocupação. A maioria possui algum transtorno mental e não são reincidentes. 0 método mais utilizado é o envenenamento por medicamentos. Esses dados demonstram a necessidade de intervenções junto a essa parcela da sociedade com vistas à promoção da saúde mental, identificação precoce dos casos por meio de ações intersetoriais e ainda mobilização social, com vistas à uma efetiva implementação das políticas públicas em favor do controle de tal agravo.
\end{abstract}

Palavras-chave: Tentativa de Suicídio; Epidemiologia; Perfil de Saúde

\section{Abstract}

The suicide is considered a human phenomenon in ascension, which is complex and universal issue that happens in every society. Suicide is a serious healthy public problem, nonetheless, one can be avoided. The goal of this study was describe the profile of people who tried to commit suicide in the city of Monte Carmelo MG. This article is about a descriptive epidemiological study, which data about the attempting of suicide was found by the compulsory notification files of exogenous intoxication and violence interpersonal and self-induced of SINAN/MS in the period of January to December of 2018. The 78 people who tried to commit suicide are most female aged under 20 to 40 years old. They most studied up to the high school, single as marital status, brown-skinned, unemployed or with no occupation. The majority of them have some perturbation, but they have not attempted suicide before, and the method that is most used by them is poisoning by medications. These data show the need of interventions along with the help of the society, aiming to promote mental health, the premature identification of the cases by intersectional actions and social mobilization, with the eye on an effective implementation of the public politics in favor of the control of this disease.

Keywords: $\quad$ Attempting of Suicide. Epidemiology. Health Profile. 


\section{INTRODUÇÃO}

O suicídio é considerado um fenômeno humano, complexo e universal, por ocorrer em todas as sociedades (MINAYO, 2005 apud MORGADO, 2006). A pessoa que pensa em suicídio ou tenta se matar está sofrendo e quando a percepção desse sofrimento se torna insuportável, ela não encontra uma maneira de amenizar ou compreender essa situação, o suicídio passa ser a única opção (CASSORLA, 2018).

O termo "suicídio", de acordo com Cassorla, (2018) significa "morte de si mesmo". Essa definição parece simples, contudo quando analisamos os vários fatores que envolvem esse fenômeno e sobre as formas como ele se manifesta, nota-se que essa conceituação é mais complexa do que parece ser, pois envolve atos e comportamentos que normalmente não são associados ao suicídio, mas que de alguma maneira se relaciona com ele, como por exemplo, a pessoa que fuma, dirige velozmente, pratica roleta russa, entre outras situações que denota a manifestação inconsciente de aspectos autodestrutivos (CASSORLA, 2018).

Segundo a Organização Mundial de Saúde (OMS) (2000) mais de 800.000 pessoas morrem por ano vítimas de suicídio, a cada 40 segundos, uma pessoa se suicida no mundo e esse número pode chegar a 1,6 milhão em 2020. Nas Américas o risco de morrer por suicídio é estimado em 6.1/100.000 habitantes. Apesar de alarmantes os números podem estar subestimados em 20 vezes em virtude da subnotificação ou inexistência de registros em países da África e Oriente Médio. Essa organização elencou o suicídio entre suas prioridades e ressalta a importância de sua prevenção, para tal, elaborou um plano de ação em saúde mental que pretende reduzir esses índices em 10\% no período de 2013 a 2020 (WHO, 2014).

A abrangência de uma morte em decorrência de suicídio vai além do esperado, ao menos seis pessoas próximas à vítima sofrem danos em aspectos emocionais, sociais e econômicos. Tal situação pode ser agravada ao passo que segundo a OMS (2000), 75\% dos casos de suicídio ocorrem em países subdesenvolvidos ou em desenvolvimento, sinalizando uma correlação entre situação econômica e taxas de suicídio, apesar de algumas exceções como Grécia, Coréia do Sul e do Norte, o que soma a inferência de fatores culturais e políticos (FERREIRA JÚNIOR, 2015).

O suicídio pode ser analisado sob diversos aspectos, como filosófico, sociológico, antropológico, moral, religioso, biológico, bioquímico, histórico, econômico, estatístico, legal, psicológico, psicanalítico, ente outros. Em partes estes aspectos estão interligados (CASSORLA, 2018).

O Brasil é um país que apresenta baixas taxas de suicídio e tentativa de suicídio, em número de ocorrências. A Taxa de suicídio, segundo a OMS (2014) é de 5,8 por 100.000 habitantes, sendo que entre homens $(9,4)$ é maior em relação às mulheres $(2,5)$.

Geograficamente, no período de 2002 a 2012, no Brasil, a região norte apresenta um crescimento expressivo nas taxas de suicídio, alcançando um aumento de 77,7\%. Os estados Acre, Roraima, Tocantins e Amazonas duplicaram os casos. A região nordeste apresentou nesse período aumento de 51,7\%, em especial os estados Paraíba e Bahia. As regiões Centro oeste sofrem aumento de $16,3 \%$ e a região Sul de 15,2. A região Sudeste sofre um incremento de 35,7 com destaque para o estado de Minas Gerais que sofre um aumento de $58,3 \%$ nos casos de morte por suicídio (WAISELFISZ, 2014, apud FERREIRA JUNIOR, 2015).

No Brasil as taxas de mortalidade apresentam discreto incremento, contudo em algumas cidades e entre a população jovem masculina apresentam taxas elevadas. Apesar desse crescimento os dados ainda sofrem interferência moral, religiosa, cultural e imprecisão no preenchimento dos dados o que resulta em subnotificação (MINAYO, 2005 apud MORGADO, 2006). Ressalta-se que a real situação não é conhecida, posto que, para cada caso notificado existam pelo menos 4 que não são registradas (VIDAL et al., 2013).

O suicídio é considerado um problema de saúde pública, por afetar a saúde individual e coletiva (OMS, 2000). Com esse objetivo o Ministério da saúde por meio da Portaria de Consolidação 2/2017, capítulo I, Seção II, artigo $3^{\circ}$, inciso III na forma do anexo VIII, apresenta Diretrizes Nacionais para Prevenção do Suicídio, com origem na PRT MS/GM 1876/2006 e preconiza entre outras ações, a articulação entre órgãos governamentais e não governamentais, desenvolvimento de estratégias de promoção da qualidade de vida, educação, proteção e recuperação da saúde, prevenção de danos, informação, comunicação e sensibilização da sociedade de que o suicídio é um problema de saúde pública que pode ser prevenido além de uma linha de cuidados em todos os níveis de atenção, garantindo o acesso às diferentes modalidades terapêuticas.

Ressalta-se a identificação da prevalência dos determinantes e condicionantes do suicídio e tentativas, assim como os fatores protetores e o desenvolvimento de ações intersetoriais de responsabilidade pública, sem excluir a responsabilidade de toda a sociedade (BRASIL, 2017). Muitas são as reflexões que se preocupam em superar os obstáculos, contudo a efetivação junto aos profissionais da área da saúde, ainda é um desafio.

A OMS destaca como fatores que fornecem proteção contra o comportamento suicida padrões familiares estáveis, boas relações sociais, capacidade de procurar ajuda ou conselhos frente a dificuldades, fatores culturais, de integração social, lazer entre outros. As situações de risco são várias e envolvem fatores 
ambientais e genéticos e são dependentes de fatores culturais, políticos e econômicos que diferem mesmo entre países vizinhos (OMS, 2000).

O Suicídio é um mal que pode ser evitado, o sofrimento pode se tornar suportável se a pessoa puder contar com a ajuda de profissionais capacitados para enfrentar esse problema. Cassorla (2018), diz que o profissional utilizará seu conhecimento para compreender esse malestar e ajudar a transformá-lo, e essa transformação permite que a pessoa reveja sua vida.

0 presente estudo foi realizado a partir do questionamento sobre o perfil da pessoa que tenta suicídio no município de Monte Carmelo-MG, com vistas a servir de subsídio para o planejamento de futuras intervenções em promoção de saúde mental junto à rede de atenção psicossocial deste município.

o Objetivo deste estudo foi descrever o perfil das pessoas que tentaram suicídio no município de Monte Carmelo MG.

\section{METODOLOGIA}

Trata-se de um estudo epidemiológico descritivo cujos dados sobre tentativa de suicídio foram obtidos por meio das fichas de notificação compulsória de intoxicação exógena e violência interpessoal / autoprovocada do SINAN do Ministério da Saúde/Departamento de Análise e Tabulação de Dados do Sistema Único de Saúde (DATASUS).

As variáveis destacadas foram sexo, idade, escolaridade, estado civil, raça, ocupação, presença de transtornos mentais, reincidência, motivação e meio utilizado. 0 período de estudo corresponde de janeiro a dezembro do ano de 2018.

O município de Monte Carmelo localiza-se na região do Triângulo Mineiro, possui IDHM de 0,728 e uma população estimada de 47.682 habitantes em 2018 (IBGE, 2019). A economia é baseada na atividade de indústria cerâmica e na agricultura com o plantio do café.

Os dados foram analisados utilizando frequência simples absoluta e em porcentagem e para o cálculo da taxa morbidade foram considerados no numerador o número de tentativas de suicídio que levaram a pessoa a ser atendida no Pronto Socorro Municipal (ficha de notificação do SINAN) e no denominador a população do município conforme IBGE.

\section{RESULTADOS E DISCUSSÃO}

No decorrer do ano de 2018 ocorreram 78 casos, o que resulta no risco de tentativa de suicido em Monte
Carmelo de 1,6/1000 habitantes. Quanto ao perfil sócio demográfico, as pessoas que tentaram suicídio no período do estudo são do sexo feminino 55 (70,6\%), com idades menores de 20 anos de idade 25 (32\%), ensino fundamental ou médio completos 18 cada (46\%), solteiras 38 (48,7\%), pardas 55 (70,5\%), desempregadas ou sem ocupação 22 (28,3\%) conforme demonstra a Tabela 1.

O suicídio parece ser mais comum entre homens e a tentativa entre mulheres (OMS, 2000). 0 que corrobora com os achados desse estudo. Entre os fatores potenciais para essa diferença são apontadas as questões de igualdade de gênero, diferenças sociais de lidar com conflitos e estresse, disponibilidade e preferência aos diferentes meios para o suicídio, consumo de álcool, acesso e procura por tratamento para os transtornos mentais (WHO, 2014). Em estudo sobre ideação, planejamento e tentativa de suicídio a prevalência de ideação suicida foi mais frequente entre mulheres (BOTEGA et al., 2009).

Em tentativa de suicídio em uma cidade do interior da Bahia observou-se o predomínio do sexo feminino $(53,4 \%)$ ao passo que suicídios as mais prevalentes entre os homens $(87,5 \%)$ (SOUZA et al., 2011). No Rio de Janeiro, $68 \%$ da amostra eram do sexo feminino (WERNECK et al., 2006). Em Minas Gerais no período de 1998 a 2003 as internações por tentativa de suicídio foram em sua maioria $(55,4 \%)$ por pessoas do sexo masculino (ABASSE et al., 2009).

Quanto a idade a distribuição de casos de tentativa de suicídio neste estudo tem predominância entre jovens de menores de 20 até 40 anos de idade. Nesta faixa etária ocorreram 58 casos (74,3\%), dentre esses, os menores de 20 anos de idade totalizam 25 (43,1\%) casos.

A ocorrência da tentativa de suicídio em faixas etárias cada vez menores é preocupante ao passo que quanto mais jovem maior a vulnerabilidade social e emocional. Conforme a Organização Mundial de Saúde (2014), em um terço dos países tanto nos desenvolvidos quanto nos em desenvolvimento observa-se essa tendência a qual será mantida nos próximos anos e destaca ainda, que o suicídio é a segunda causa de morte entre jovens (WHO, 2014).

Em estudo sobre ideação, planejamento e tentativa de suicídio a prevalência de ideação suicida foi mais frequente entre adultos de 30 a 44 anos (BOTEGA et al., 2009). Nos casos de suicídio no interior da Bahia houve maior concentração na faixa etária de 40 anos e mais, ao passo que as tentativas ocorreram na faixa etária de 20 a 39 anos (SOUZA et al., 2011). No Rio de Janeiro 26\% eram adolescentes (WERNECK et al., 2006).

No aspecto referente a escolaridade, destaca-se 15 pessoas com ensino fundamental incompleto, $18 \mathrm{com}$ fundamental completo, 17 com médio incompleto, 18 
Tabela 1 - Perfil sócio demográfico: tentativas de suicídio, Monte Carmelo - MG, janeiro a dezembro de 2018.

\begin{tabular}{|c|c|c|c|}
\hline \multicolumn{2}{|c|}{ Variável } & \multirow{2}{*}{$\begin{array}{l}\mathrm{N} \\
23 \\
55\end{array}$} & \multirow{2}{*}{$\begin{array}{c}\% \\
29,4 \\
70,6\end{array}$} \\
\hline Sexo & $\begin{array}{l}\text { Masculino } \\
\text { Feminino }\end{array}$ & & \\
\hline Idade & $\begin{array}{c}<20 \\
20-30 \\
30-40 \\
40-50 \\
50-60 \\
>60\end{array}$ & $\begin{array}{c}25 \\
18 \\
15 \\
14 \\
5 \\
1\end{array}$ & $\begin{array}{c}32,0 \\
23,0 \\
19,3 \\
17,9 \\
6,5 \\
1,3\end{array}$ \\
\hline Escolaridade & $\begin{array}{c}\text { Analfabeto } \\
\text { Primário Completo } \\
\text { Primário Incompleto } \\
\text { Fundamental Incompleto } \\
\text { Fundamental Completo } \\
\text { Médio Incompleto } \\
\text { Médio Completo } \\
\text { Superior Incompleto } \\
\text { Ignorado }\end{array}$ & $\begin{array}{c}1 \\
4 \\
2 \\
15 \\
18 \\
17 \\
18 \\
1 \\
2\end{array}$ & $\begin{array}{r}1,3 \\
5,1 \\
2,6 \\
19,3 \\
23,0 \\
21,8 \\
23,0 \\
1,3 \\
2,6\end{array}$ \\
\hline Estado Civil & $\begin{array}{l}\text { Casado } \\
\text { Solteiro } \\
\text { Separado } \\
\text { Ignorado }\end{array}$ & $\begin{array}{c}25 \\
38 \\
4 \\
11\end{array}$ & $\begin{array}{c}32,0 \\
48,7 \\
5,1 \\
14,2\end{array}$ \\
\hline Raça & $\begin{array}{l}\text { Branca } \\
\text { Parda } \\
\text { Negra }\end{array}$ & $\begin{array}{c}19 \\
55 \\
4\end{array}$ & $\begin{array}{c}24,4 \\
70,5 \\
5,1\end{array}$ \\
\hline Ocupação & $\begin{array}{c}\text { Sem/Desempregado } \\
\text { Estudante } \\
\text { Do Lar } \\
\text { Rural } \\
\text { Aposentado } \\
\text { Vendedor } \\
\text { Outras } \\
\text { Ignorado }\end{array}$ & $\begin{array}{c}22 \\
14 \\
12 \\
5 \\
3 \\
3 \\
5 \\
14 \\
\end{array}$ & $\begin{array}{c}28,3 \\
17,9 \\
15,5 \\
6,4 \\
3,8 \\
3,8 \\
6,4 \\
17,9 \\
\end{array}$ \\
\hline
\end{tabular}

Fonte: Fichas de notificação SINAN, Violência Interpessoal / Auto Provocada e Intoxicação Exógena, janeiro a dezembro, Monte Carmelo, 2018.

com médio completo, totalizando nesses níveis de escolaridade a maioria da amostra, 68 pessoas $(87,1 \%)$.

A escolaridade apesar da dificuldade encontrada pelos autores no estudo realizado no interior da Bahia, resultante do não preenchimento do campo pertinente no formulário utilizado, foi encontrado como característica das pessoas que tentaram suicídio 4 a 7 anos de estudos concluídos (SOUZA, et al., 2011).

Em relação ao estado civil prevaleceu entre os que tentaram suicídio neste estudo os solteiros (48,7\%) em relação aos casados, assim como no estudo realizado no interior da Bahia por Souza (2011). No Rio de Janeiro $27 \%$ eram casados ou vivendo em união estável (WERNECK et al., 2006).

Quanto ao aspecto raça/cor, houve predomínio entre os que tentaram suicídio neste estudo, dos que se consideram pardos totalizando 55 pessoas (68\%). Entre as pessoas que tentaram suicídio em um município no interior da Bahia, a maioria eram pardas, seguidas por brancas e pretas (SOUZA et al., 2011).

Quanto à ocupação a maioria 22 (28,2\%) estava desempregada ou não tinham ocupação. Entre os demais $14(17,9 \%)$ eram estudantes, ignorados 14 $(17,9 \%)$, do lar $12(15,3 \%)$, trabalhador rural 5(6,4\%), aposentados $3(3,8 \%)$, vendedores $3(3,8 \%)$, citados ainda com $n=1$ as ocupações de pintor, vigilante, soldador, serviços gerais, cabeleireira e mecânico. No interior da Bahia foram identificadas ocupações relatadas entre os que tentaram suicídio, o predomínio de estudantes seguidos de profissionais autônomos, com destaque para carpinteiros, comerciantes, trabalhadores rurais. Com menor percentual, agrônomo, aposentado, do lar, funcionário público, industriário, motorista e pedreiro (ZOUZA, et al., 2011). No Rio de Janeiro houve predomínio de estudantes, do lar e aposentadas entre mulheres e entre os homens, 
desempregados e trabalhadores manuais (WERNECK et al., 2006).
Quanto aos dados sobre presença de transtorno mental e reincidência, estes foram agrupados na Tabela 2.

Tabela 2 - Presença de Transtorno Mental e Reincidência entre Tentativas de Suicídio, Monte Carmelo, MG, 2018.

\begin{tabular}{|c|c|c|c|}
\hline \multicolumn{2}{|c|}{ Variável } & $\mathrm{N}$ & $\%$ \\
\hline \multirow{3}{*}{$\begin{array}{c}\text { Presença de Transtorno Mental } \\
\text { Ignorados }\end{array}$} & Sim & 36 & 46,1 \\
\hline & Não & 29 & 37,2 \\
\hline & & 13 & 16,7 \\
\hline & Sim & 31 & 39,8 \\
\hline \multirow[t]{2}{*}{ Reincidente } & Não & 39 & 50 \\
\hline & Ignorado & 8 & 10,2 \\
\hline
\end{tabular}

Fonte: Fichas de notificação SINAN, Violência Interpessoal / Auto Provocada e Intoxicação Exógena, janeiro a dezembro, Monte Carmelo, 2018.

Houve predomínio de pessoas com transtorno mental $36(46,1 \%)$ e que tentaram se matar pela primeira vez. Os fatores de risco para suicídio são vários dentre eles destaca-se tentativas anteriores que são consideradas como o principal fator preditivo. O risco aumenta de acordo com o número de tentativas anteriores e intervalos menores ente elas. Estima-se que para cada de suicídio existam pelo menos 10 tentativas que levariam ao atendimento médico (VIDAL et al., 2013).

Suicídio e comportamento suicida são mais comuns em pessoas com algum transtorno psiquiátrico, os grupos diagnósticos mais comuns em ordem decrescente de risco são depressão, transtornos de personalidade com destaque para o antissocial e borderline, alcoolismo, esquizofrenia e transtornos mentais orgânicos (OMS, 2000).

Entre os fatores associados à ideação e tentativas de suicídio destaca-se os transtornos psiquiátricos (BOTEGA et al., 2009).
Ressaltam-se transtornos de personalidade transtornos mentais orgânicos e algumas doenças físicas (OMS, 2000).

Quanto à motivação, conflito geracional somaram 49 (62,9\%), transtorno mental $6(7,7 \%)$ e ignorados 23 (29.5\%). Apesar desse estudo não ter como objetivo aprofundar na determinação da causa das tentativas de suicídio, vale ressaltar que por associação corroboramos com estudo anterior, no qual a autora afirma que: "uma parcela reduzida das tentativas de suicídio possuiam o desejo de se matar, o ato é marcado por ambivalência em ralação a viver ou morrer, o que reforça a ideia de um pedido de ajuda" (BOTEGA et al., 2009).

O método mais utilizado para as tentativas de suicídio neste estudo foi o envenenamento $70(89,7 \%)$, que ocorreu em sua maioria $47(67,1 \%)$ com medicamentos, conforme demonstra a Tabela 3.

Tabela 3 - Método utilizado para Tentativas de Suicídio, Monte Carmelo - MG, 2018.

\begin{tabular}{|c|c|c|c|}
\hline \multicolumn{2}{|c|}{ Método } & N. & $\%$ \\
\hline \multirow{4}{*}{ Técnica / Método utilizado } & Envenenamento & 70 & 89,7 \\
\hline & Pérfuro-Cortante & 4 & 5,1 \\
\hline & Enforcamento & 2 & 2,6 \\
\hline & Objeto Contundente & 1 & 1,3 \\
\hline \multirow{7}{*}{ Grupo } & Ignorado & 1 & 1,3 \\
\hline & Medicamentos & 47 & 67,1 \\
\hline & Inseticida/Raticida & 4 & 5,7 \\
\hline & Água Sanitária & 2 & 2,9 \\
\hline & Agrotóxico & 6 & 8,6 \\
\hline & Tinta & 1 & 1,4 \\
\hline & Ignorado & 10 & 14,3 \\
\hline
\end{tabular}

Fonte: Fichas de notificação SINAN, Violência Interpessoal / Auto Provocada e Intoxicação Exógena, janeiro a dezembro, Monte Carmelo, 2018.

Em Minas Gerais, as internações por tentativa de suicídio, no período de 1998 a 2003 entre adolescentes, o método de auto intoxicação foi o mais utilizado em ambos os sexos, com prevalência três vezes maior entre as mulheres, seguido por lesões decorrentes de objeto pérfuro-cortantes ou penetrantes. (ABASSE et al., 2009). 
No Rio de Janeiro houve a prevalência de tentativas de suicídio com a ingestão de pesticidas (52\%) e medicamentos (39\%) (WERNECK et al., 2006).

\section{CONCLUSÕES}

O perfil das pessoas que tentaram suicídio no município de monte Carmelo, quanto aos fatores sócio demográficos, são pessoas do sexo feminino, com idades entre menores de 20 e 40 anos de idade, com formação até o ensino médio, solteiras, pardas, desempregadas ou sem ocupação. A maioria possui algum transtorno mental e não são reincidentes. 0 método mais utilizado é o envenenamento por medicamentos.

Esses dados demonstram a necessidade de intervenções junto a essa parcela da sociedade com vistas à promoção da saúde mental, identificação precoce dos casos por meio de ações intersetoriais e ainda a mobilização social, com vistas à uma efetiva implementação das políticas públicas em favor do controle de tal agravo.

\section{REFERÊNCIAS}

ABASSE, M. L. F. et al. Análise epidemiológica da morbimortalidade por suicídio entre adolescentes em Minas Gerais, Brasil. Ciência e Saúde Coletiva, v. 14, n. 2, p. 407 416, 2009.

BOTEGA, N. J. et al. Prevalências de ideação, planos e tentativas de suicídio: um inquérito populacional em Campinas SP. Cadernos de Saúde Pública, v. 25, n. 12, p. 2632-2638, 2009.

BRASIL. Ministério da Saúde. Gabinete do Ministro. Portaria $\Pi^{\circ} 02$ de 28 de setembro de 2017. Regulamenta a consolidação das normas sobre as políticas nacionais de saúde do Sistema Único de Saúde. Disponível em: http://bvsms.saude.gov.br/bvs/saudelegis/gm/2017/prc0002 0310 2017.html. Acesso em 10/05/2019.

CASSORLA, Roosevelt Moises Smeke. Suicídio: fatores inconscientes e aspectos socioculturais. São Paulo: Blucher, 2018, $112 p$

FERREIRA JUNIOR, Avimar. O comportamento suicida no Brasil e no mundo. Revista Brasileira de Psicologia, v. 2, n. 1. 2015.

IBGE - Instituto Brasileiro de geografia e estatística. Estimativa populacional. Disponível em: https://cidades.ibge.gov.br/brasil/mg/montecarmelo/panorama. Acesso em 10/05/2019.
LOVISI, G. M. et al. Análise epidemiológica do suicídio no Brasil entre 1980 e 2006. Revista Brasileira de Psiquiatria, v. 31(supl), p. 86-93, 2009.

MINAYO, M. C. S. Impacto da violência na saúde dos brasileiros. Ministério da Saúde, 2005. 340 p. Brasília: Ministério da Saúde. Resenha de: MORGADO, Rosana. Impacto da violência na saúde dos brasileiros. Ciência \& Saúde Coletiva, v. 11, n. 2, p.537-540, 2006

ORGANIZAÇÃO MUNDIAL DE SAÚDE. Prevenção do

Suicídio: Manual para Professores e Educadores. Genebra, 29p., 2000. Disponível em:

https://apps.who.int/iris/bitstream/handle/10665/66801/W HO MNH MBD 00.3 por.pdf;jessionid=41ADC27EBAF64B 8DA80F3221C32D6E6A? sequence $=5$. Acesso em $10 / 05 / 2019$

ORGANIZAÇÃO MUNDIAL DE SAÚDE, Prevenção Do Suicídio: Um Manual para Profissionais da Saúde em Atenção Primária. Genebra, 22p., 2000. Disponível em: https://www.who.int/mental health/prevention/suicide/en/s vicideprev phc port.pdf. Acesso em 10/05/2019.

SOUZA, V. dos S. et al. Tentativas de suicídio e mortalidade por suicídio em um município no interior da Bahia. Jornal Brasileiro de Psiquiatria. v. 60, n. 4, p. 294-300, 2011.

VIDAL, C. E. L.; GONTIJO, E. C. D. M.; LIMA, L. A.; Tentativas de suicídio: fatores prognósticos e estimativa do excesso de mortalidade. Cadernos de Saúde Pública, Rio de Janeiro, 29(1):175-187, jan, 2013.

WERNECK, G. L. et al.Tentativas de suicídio em um hospital geral no Rio de Janeiro, Brasil. Cadernos de Saúde Pública, Rio de Janeiro, v. 22, n. 10, p. 2201-2206, 2006.

WORLD HEALTH ORGANIZATION. Preventing suicide: a global imperative. Geneva. 2014. Disponível em: https://apps.who.int/iris/bitstream/handle/10665/131056/9 789241564779 eng.pdf;jsessionid=A1DC7D7E91C3D6CBOC C2FFE8A81DA680? sequence $=1$. Acesso em 10/05/2019. 